Preprints of the

Max Planck Institute for

Research on Collective Goods

Bonn 2012/22

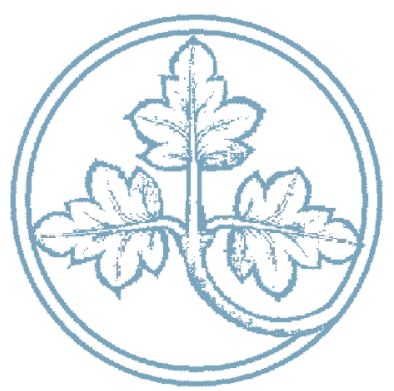

The Dynamics of Utility in the Neoclassical OLG Model

Wolfgang Kuhle

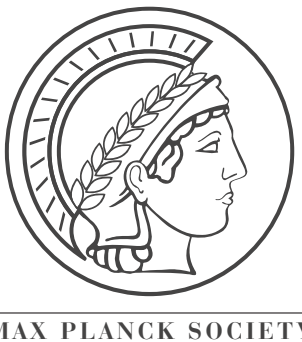




\section{The Dynamics of Utility in the Neoclassical OLG Model}

Wolfgang Kuhle

November 2012 


\title{
The Dynamics of Utility in the Neoclassical
}

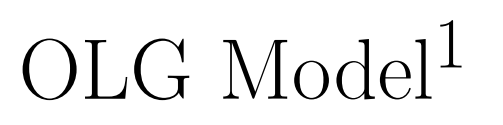

\author{
Wolfgang Kuhle \\ Max Planck Institute for Research on Collective Goods, Kurt-Schumacher-Str. 10, 53113 \\ Bonn, Germany. Email: kuhle@coll.mpg.de.
}

\begin{abstract}
This paper develops a method to study how life-cycle utility of a sequence of cohorts converges towards its steady state level in the neoclassical two-generations-overlapping model. This method allows to characterize utility changes associated with variations in exogenous policy parameters along the entire transition path between two steady states. At the same time it is not more complicated than a pure steady state analysis. Moreover, it can be applied to economies for which an explicit solution of the transition path is not available.
\end{abstract}

\section{Keywords: Overlapping Generations, Transition Path, Utility Dynamics JEL: E13}

\section{Introduction}

The normative evaluation of the neoclassical overlapping generations model has been mainly concerned with comparisons of steady state paths which are associated with varying exogenous parameter values. Unfortunately, such an analysis neglects the transitional dynamics and thus leaves out those cohorts that live during the infinite time-span which elapses before a steady state is reached. This negligence of transitional phenomena is particularly unfortunate since the main strength of OLG models, i.e., the explicit intertemporal structure of trades, is compromised when attention is confined to steady states in which a young cohort overlaps only with an older, but otherwise identical, cohort. Moreover, those economies which allow to solve for transition paths explicitly require restrictions on preferences and technology which make it difficult to understand whether these findings carry over to more general specifications.

The present paper develops a simple method to characterize the dynamics of utility along the transition path between two competitive steady states. The dynamics of utility will be

\footnotetext{
${ }^{1}$ I thank Sophie Bade and Dominik Grafenhofer for detailed comments which helped me to improve this paper. I also thank Carl Christian von Weizsäcker for a discussion on transition paths. First version November 2011. Revised June 2012.
} 
described by a first-order difference equation in life-cycle utility $U_{t}$. This equation describes how life-cycle utilities $\left\{U_{t}\right\}$ obtained by a sequence of cohorts that live along the transition path are interrelated. The advantage of the current approach is that it can be applied to economies for which an explicit solution of the transition path is not available. For such economies, it is a useful device to evaluate the utility implications of one-time permanent policy changes as it allows to compare utility along the transition path to the old steady state allocation which would have obtained had the exogenous parameter remained unchanged. That is, the present method can be used to extend previous normative studies which perform only steady state comparisons: it adds the complete transition path, and therefore allows to Paretoevaluate parameter changes ${ }^{2}$ Moreover, while it characterizes the complete transition path, it is analytically not more complicated than the familiar steady state comparison which is contained as a limiting case.

The paper is organized as follows: In section 2 we characterize for given parameter values how utility evolves over time in an economy which is initially not in a steady state. In particular, we show that the standard existence and uniqueness conditions for steady state equilibria by Galor and Ryder (1989) and de la Croix and Michel (2002) imply that utility converges monotonically towards its steady state value. In section 3 we show how changes in utility along the transition path between two steady states, which are induced by a permanent parameter change, can be characterized. In section 4 we apply the method developed in section 3 to the original Diamond (1965) problem of national debt to illustrate how the present approach provides new insights into the dynamic properties of this economy. The original framework developed by Diamond (1965) provides a useful example since it uses general utility and production functions such that an explicit solution of the transition path is not available. Moreover, this example allows us to compare the present analytical approach to the alternative approach developed by Matsuyama (1991), which is based on a geometric argument. Finally, section 5 offers concluding remarks.

\footnotetext{
${ }^{2}$ Representative studies that confine the normative analysis to steady states (and leave the transition path for "future research") are Diamond (1970), von Weizsäcker (1971), Samuelson (1975), Galor and Polemarchakis (1987), Galor (1988), or Casarico and Devillanova (2008). The Subsequent approach will allow to add the complete transition path to these studies. It will, however, not require any substantial additional calculations or assumptions.
} 


\section{The Pure Dynamics of Utility}

In this section we introduce the Diamond (1965) model in its basic from without public debt. Starting from a situation where the economy is not in a steady state, we derive a first order difference equation which has life-cycle utility $U_{t}$ as its endogenous variable. Using this difference equation we show that utility converges monotonically towards its steady state value if the economy satisfies the standard conditions derived by Galor and Ryder (1989) and de la Croix and Michel (2002), which ensure the existence of a unique steady state equilibrium with a positive capital intensity.

Production is characterized by capital and labor inputs, $K_{t}$ and $L_{t}$, which produce aggregate output $Y_{t}=F\left(K_{t}, L_{t}\right)$, where $F($,$) is concave, first-degree-homogenous and satisfies the Inada$ conditions. Output per unit of labor in period $t$ can thus be written as:

$$
y_{t}=f\left(k_{t}\right), \quad k_{t}:=\frac{K_{t}}{L_{t}}, \quad f^{\prime}\left(k_{t}\right)>0, \quad f^{\prime \prime}\left(k_{t}\right)<0 .
$$

Factor markets are competitive such that wages $w_{t}$ and interest rate $r_{t}$ are

$$
r_{t}=f^{\prime}\left(k_{t}\right), \quad w_{t}=f\left(k_{t}\right)-f^{\prime}\left(k_{t}\right) k_{t} .
$$

Population grows at rate $n$, and each cohort lives for two periods. In the first period of life each individual supplies one unit of labor inelastically. The second period of life is spent in retirement. The associated life-cycle savings problem for an individual born in period $t$ is given by:

$$
\max _{s_{t}, c_{t}^{1}, c_{t+1}^{2}} U_{t}=U\left(c_{t}^{1}, c_{t+1}^{2}\right) \quad \text { s.t. } \quad c_{t}^{1}=w_{t}-s_{t}, \quad c_{t+1}^{2}=s_{t}\left(1+r_{t+1}\right),
$$

such that the first order condition

$$
\frac{U_{c^{1}}}{U_{c^{2}}}=1+r_{t+1} \quad \Leftrightarrow \quad s_{t}=s\left(w_{t}, r_{t+1}\right), \quad 0<s_{w}<1, \quad s_{r} \gtreqless 0,
$$

defines savings. Regarding the utility function in (3), we assume that it is concave, twice continuously differentiable and that it satisfies the Inada conditions $3^{3}$ These assumptions ensure that savings in (4) are positive, unique, and continuously differentiable in its two arguments $w_{t}$ and $r_{t+1}$. Moreover, we assume that first and second period consumption are normal goods such that the propensity to save out of income satisfies $0<s_{w}<1$.

Finally, the life-cycle savings condition

$$
(1+n) k_{t+1}-s_{t}\left(w_{t}, r_{t+1}\right)=0
$$

\footnotetext{
${ }^{3}$ Namely, $U($, $)$ is such that $\lim _{c^{1} \rightarrow 0} U_{c^{1}}\left(c^{1}, c^{2}\right)=+\infty$ and $\lim _{c^{2} \rightarrow 0} U_{c^{2}}\left(c^{1}, c^{2}\right)=+\infty$.
} 
closes the model. To obtain the law of motion of the capital stock, we substitute (2) into (5) to obtain:

$$
(1+n) k_{t+1}-s_{t}\left(f\left(k_{t}\right)-f^{\prime}\left(k_{t}\right) k_{t}, f^{\prime}\left(k_{t+1}\right)\right)=0
$$

Regarding (6), we follow Galor and Ryder (1989), de la Croix and Michel (2002), and Michel and de la Croix (2000), and assume that the following two conditions, which ensure the existence of a unique steady state with a positive capital intensity, are satisfied:

$$
\begin{aligned}
& \frac{d\left((1+n) k-s\left(f(k)-f^{\prime}(k) k, f^{\prime}(k)\right)\right)}{d k}=(1+n)+s_{w} f^{\prime \prime}(k) k-s_{r} f^{\prime \prime}(k)>0, \\
& \lim _{k \rightarrow 0} \frac{s(w(k), r(k))}{k}>1+n .
\end{aligned}
$$

Combining (6) with assumption (7), we have the following lemma:

Lemma 1. The capital intensity $k_{t}$ converges monotonically to its steady state level $k$.

Proof. Differentiation of (6) at the steady state, where $k_{t+1}=k_{t}=k$, gives $\frac{d k_{t+1}}{d k_{t}}=\frac{-s_{w} f^{\prime \prime}(k)}{(1+n)-s_{r} f^{\prime \prime}(k)}$. To show that the capital stock converges monotonically to the steady state it is necessary that $0<\frac{d k_{t+1}}{d k_{t}}=\frac{-s_{w} f^{\prime \prime}(k)}{(1+n)-s_{r} f^{\prime \prime}(k)}<1$. To see that this condition is satisfied we recall $(7)$ which, together with our assumption that $0<s_{w}$ and $f^{\prime \prime}(k)<0$, implies that $0<-s_{w} f^{\prime \prime}(k)<(1+n)-s_{r} f^{\prime \prime}(k)$. Dividing both sides of this inequality by $(1+n)-s_{r} f^{\prime \prime}(k)$ yields $0<\frac{-s_{w} f^{\prime \prime}(k)}{(1+n)-s_{r} f^{\prime \prime}(k)}<1$, and completes the proof.

While Lemma 1 reflects what standard assumption (7) implies, it is well known that there is a second possibility. Namely, if first period and second period consumption are complements, we have $s_{r}<0$ and the inequality in (7) may be reversed. In this case, which we will call the Marshallian case, the capital stock converges cyclically towards the steady state equilibrium, that is, we have $-1<\frac{d k_{t+1}}{d k_{t}}<0.4^{4}$ In the following, we focus mainly on the Walrasian case described in Lemma 1 where the capital stock converges monotonically. However, in the following analysis, we will at times use the Marshallian case as a contrasting device for the results that we derive for the standard Walrasian economy.

\footnotetext{
${ }^{4}$ See Diamond (1965), p. 1132 and p. 1148, for the details on this case. In particular, Diamond (1965) shows that this case can only occur if the capital market reaches equilibrium through changes in the quantity of capital supplied (Marshallian adjustment). Accordingly, we call this case the Marshallian case. If the capital market reaches equilibrium through changes in price (Walrasian adjustment), capital must converge monotonically. Samuelson (1941), pp. 102-106, discusses the Walrasian and the Marshallian market mechanism in detail.
} 
With these observations in place we can derive our first result on the convergence of life-cycle utility:

Proposition 1. Life-cycle utility $U_{t}$ follows a continuously differentiable first order difference equation $U_{t+1}=\xi\left(U_{t}\right)$. Moreover, life-cycle utility converges monotonically to its steady state level where $U_{t}=U_{t+1}=U$ iff the capital stock converges monotonically.

Proof. By our assumptions, $U(),, s(),, f^{\prime}(), w(), r()$ are all at least once continuously differentiable $\left(\mathcal{C}^{1}\right)$ functions with non-vanishing partial derivatives. Therefore, we can ${ }^{5}$ use the implicit function theorem to define new functions $\phi()$ and $\psi()$ such that:

$$
(1+n) k_{t+1}=s\left(w\left(k_{t}\right), r\left(k_{t+1}\right)\right) \quad \Leftrightarrow \quad k_{t+1}=\psi\left(k_{t}\right), \quad \psi \in \mathcal{C}^{1},
$$

and

$$
\begin{aligned}
U_{t} & =U\left(w\left(k_{t}\right)-s\left(w\left(k_{t}\right), r\left(k_{t+1}\right)\right),\left(1+r\left(k_{t+1}\right)\right) s\left(w\left(k_{t}\right), r\left(k_{t+1}\right)\right)\right)=U\left(k_{t}, k_{t+1}\right) \\
& =\mid 99\left(k_{t}, \psi\left(k_{t}\right)\right) \Leftrightarrow \quad k_{t}=\phi\left(U_{t}\right), \quad \phi \in \mathcal{C}^{1} .
\end{aligned}
$$

Substituting (10) into (9) yields a first order difference equation in $U_{t} \cdot{ }^{6}$

$$
\phi\left(U_{t+1}\right)=\psi\left(\phi\left(U_{t}\right)\right) \quad \Leftrightarrow \quad U_{t+1}=\phi^{-1}\left(\psi\left(\phi\left(U_{t}\right)\right)\right)=: \xi\left(U_{t}\right), \quad \xi \in \mathcal{C}^{1} .
$$

With this continuously differentiable first order difference equation in place, it remains to show that utility converges monotonically iff the capital stock converges monotonically. Once we differentiate (11) around the steady state, where $U_{t+1}=U_{t}=U$, the condition for monotonous convergence is:

$$
0<\frac{d U_{t+1}}{d U_{t}}=\frac{\phi^{\prime}(U) \psi^{\prime}(\phi(U))}{\phi^{\prime}(U)}=\psi^{\prime}(\phi(U))<1 .
$$

To relate condition $(12)$ to the convergence of the capital stock, we recall that $\psi^{\prime}(\phi(U))=\mid \sqrt{10}$ $\psi^{\prime}(k)$. Hence, utility converges monotonically (cyclically) to the steady state if capital converges monotonically (cyclically), since it follows from (9) that capital converges monotonically (cyclically) if $0<\frac{d k_{t+1}}{d k_{t}}=\psi^{\prime}(k)<1\left(-1<\frac{d k_{t+1}}{d k_{t}}=\psi^{\prime}(k)<0\right)$.

\footnotetext{
${ }^{5}$ In Appendix A we show that the technical conditions which allow us to apply the implicit function theorem to derive (9), (10) and (11) are indeed satisfied. With the formalism in place, we prove a stronger result than Proposition 1. Namely, we show that $\xi()$ is a $\mathcal{C}^{1}$ diffeomorphism. That is, $\xi()$ and its inverse $\xi^{-1}()$ are both continuously differentiable. Hence, we can write $U_{t-1}=\xi^{-1}\left(U_{t}\right)$ and use current utilities $U_{t}$ to calculate past utilities $U_{t-1}$. That is, the dynamics of utility are fully symmetric with respect to time.

${ }^{6}$ By the implicit function theorem we know (Lee (2003), pp. 164-166) that $\phi^{-1} \in \mathcal{C}^{1}$ exists. Moreover, we know that $\xi \in \mathcal{C}^{1}$ since the composition of continuously differentiable functions is itself continuously differentiable. See Appendix A for details on $\phi^{-1}()$.
} 


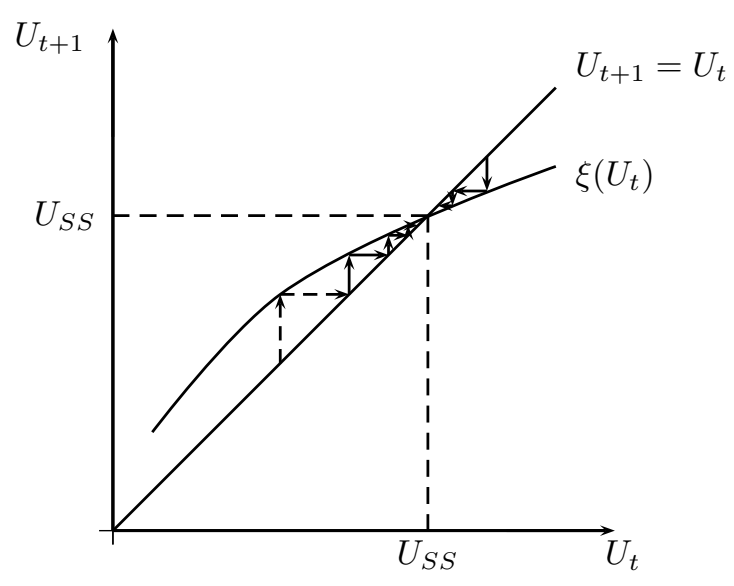

$11 a$

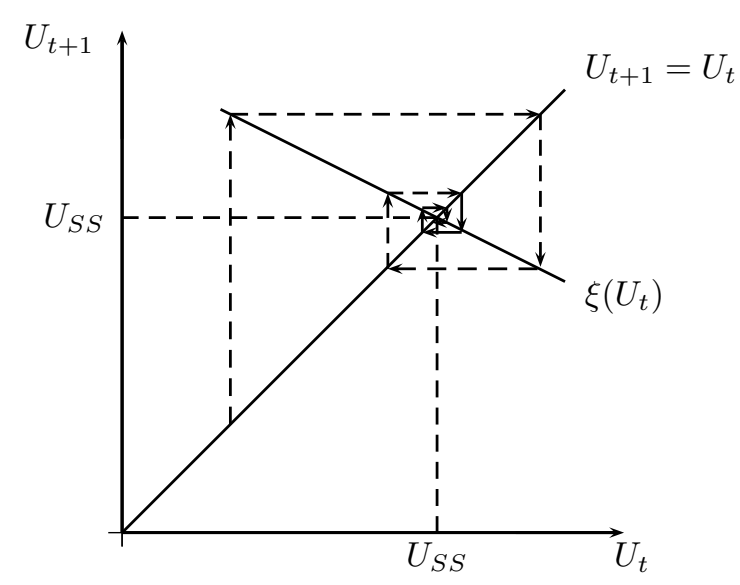

$1 b$

Diagram 1: Walrasian and Marshallian Dynamics of Utility.

Diagram 1 1 depicts the dynamics of utility characterized in Proposition 1 for the case where the capital market satisfies Walrasian stability. Diagram $1 p$ illustrates the cobweb behavior of utility in the alternative Marshallian case. The attractive property of Proposition 1 lies in the fact that it allows to draw on the rich knowledge which is available on the dynamics of the capital stock in order to understand the implied changes in utility. In particular, recalling Lemma 1, we find that the standard existence and uniqueness conditions for steady states derived by Galor and Ryder (1989) and de la Croix and Michel (2002) imply that utility converges monotonically to its steady state value. We show in section 4 that it will depend on the sign of $\psi^{\prime}(k)$ as well as the speed of convergence, i.e., the size of $\left|\psi^{\prime}(k)\right|$, whether a given change in an exogenous parameter like public debt increases or decreases utility during the period of transition.

However, currently Proposition 1 only describes the evolution of utility along a transition path, which starts at a point where the economy is not in a steady state. In the next section we change this perspective and take a steady state allocation as the point of departure. The transitional dynamics will then be induced by a parameter change and the ensuing utility implications will be decomposed into a cumulative component, an incremental component, and a direct effect.

\section{Parameter Changes}

To fully evaluate the normative implications of a change in one exogenous (policy) parameter in the present intertemporal setting we have to compare life-cycle utilities $\left\{\tilde{U}_{t}\right\}$ along the tran- 
sition path to utilities $\left\{U_{t}\right\}$ in the steady state, which would have obtained had the parameter remained unchanged. To evaluate such a policy change we augment our model (9)-(10) and write:

$$
\begin{aligned}
& k_{t+1}=\psi\left(k_{t} ; b\right), \quad 0<\psi_{k}(k ; b)<1, \quad \psi \in \mathcal{C}^{1}, \\
& U_{t}=U\left(k_{t}, k_{t+1} ; b\right),
\end{aligned}
$$

where $b$ represents the exogenous variable of interest. At this point we introduce $b$ in the most general way and allow it to affect utility directly and indirectly through its influence on the law of motion of the capital stock. Moreover, we focus on the Walrasian economy where the capital market is monotonically stable. Finally, to work with $(13)$ and (14) it is convenient to assume that a change in parameter $b$ in period 0 will affect capital from period 1 onwards but not in period 0.7 Taken together, (13) and (14) have the following implications:

Proposition 2. If the economy is initially in a steady state, a marginal change in the exogenous parameter $b$ which is enacted in period 0 changes utility of a cohort born in period $t \geqq-1$ according to

$$
\begin{aligned}
\frac{d U_{-1}}{d b} & =\frac{\partial U_{-1}}{\partial b} \\
\frac{d U_{t}}{d b} & =\frac{\partial U}{\partial k_{t}} \frac{d k_{t}}{d b}+\frac{\partial U}{\partial k_{t+1}} \frac{d k_{t+1}}{d b}+\frac{\partial U}{\partial b} \\
& =\frac{d U}{d k}\left(\sum_{i=0}^{t-1} \psi_{k}(k ; b)^{i}\right) \psi_{b}(k ; b)+\frac{\partial U}{\partial k_{t+1}} \psi_{k}(k ; b)^{t} \psi_{b}(k ; b)+\frac{\partial U}{\partial b}, \quad t \geqq 0,
\end{aligned}
$$

where $\frac{d U}{d k}:=\frac{\partial U}{\partial k_{t}}+\frac{\partial U}{\partial k_{t+1}}$.

The steady state change in utility is given by:

$$
\frac{d U_{s s}}{d b}=\lim _{t \rightarrow \infty} \frac{d U_{t}}{d b}=\frac{d U}{d k} \frac{1}{1-\psi_{k}(k ; b)} \psi_{b}(k ; b)+\frac{\partial U}{\partial b} .
$$

Proof. We begin by noting that the linearization in (16) follows from the definition of $U_{t}$ in 14 , and that the derivatives $\frac{\partial U}{\partial k_{t}}, \frac{\partial U}{\partial k_{t+1}}, \frac{\partial U}{\partial b}$ are evaluated at the initial steady state where $k_{t}=k_{t+1}=k$. To derive the key formula (17), we have to find $\frac{d k_{t}}{d b}$, which is the cumulative marginal change in period $t$ capital, which is associated with a marginal change of $b$ in period

\footnotetext{
${ }^{7}$ In the present context this assumption helps to keep notation short and can easily be relaxed (by adding one partial derivative). As we will see in the example in section 4 , this assumption is in line with most cases treated in the literature.
} 
0. Recalling (13) we write:

$$
\begin{aligned}
& k_{1}=\psi\left(k_{0} ; b\right) \\
& k_{2}=\psi\left(k_{1} ; b\right)=\psi\left(\psi\left(k_{0} ; b\right) ; b\right) \\
& \text {. } \\
& \text { · } \\
& k_{t}=\psi\left(\psi\left(\psi\left(,,, \psi\left(k_{0} ; b\right) ; ; b\right) ; b\right) ; b\right)=\psi^{(t)}\left(k_{0} ; b\right),
\end{aligned}
$$

such that repeated use of the chain rule yields the cumulative change in period $t$ capital $k_{t}$, which originates from a change of $b$ in period 0 :

$$
\frac{d k_{t}}{d b}=\sum_{i=0}^{t-1} \psi_{k}\left(k_{0} ; b\right)^{i} \psi_{b}\left(k_{0} ; b\right)=\frac{1-\psi_{k}\left(k_{0} ; b\right)^{t}}{1-\psi_{k}\left(k_{0} ; b\right)} \psi_{b}\left(k_{0} ; b\right) .
$$

Setting the initial capital stock $k_{0}=k$, substituting (20) into (16), and using the definition $\frac{d U}{d k}:=\frac{\partial U}{\partial k_{t}}+\frac{\partial U}{\partial k_{t+1}}$ yields 17 .

Next, we show that the limit in (18) indeed captures the complete steady state change in utility. To this end, we recall (13) and (14) to calculate the steady state effect directly. Differentiation of (13) and (14) at the point where $k_{t}=k_{t+1}=k$ shows that the steady state effects are

$$
\begin{aligned}
\frac{d k}{d b} & =\frac{1}{1-\psi_{k}(k ; b)} \psi_{b}(k ; b), \\
\frac{d U}{d b} & =\left(\frac{\partial U}{\partial k_{t}}+\frac{\partial U}{\partial k_{t+1}}\right) \frac{d k}{d b}+\frac{\partial U}{\partial b} \\
& =\frac{d U}{d k} \frac{1}{1-\psi_{k}(k ; b)} \psi_{b}(k ; b)+\frac{\partial U}{\partial b},
\end{aligned}
$$

where 23 follows from (21) and the definition $\frac{d U}{d k}:=\frac{\partial U}{\partial k_{t}}+\frac{\partial U}{\partial k_{t+1}}$. Most importantly, 23) is identical to the limit in 18 , which is what we needed to show.

Finally, we note that the series in (18) converges to a geometric series as $t$ goes to infinity. The geometric series converges in turn, since $0<\psi_{k}(k ; b)<1$. Moreover, after period $t=1$ we have $\frac{d U_{t+1}}{d b}-\frac{d U_{t}}{d b}=\psi_{k}^{t} \psi_{b}\left(\frac{d U}{d k}+\frac{\partial U}{\partial k_{t+1}}\left(\psi_{k}-1\right)\right)$, and, hence, marginal utility converges, just as described in Proposition 1, monotonically since we have $0<\psi_{k}<1$ by assumption (13).

Equation (17) describes the linearized impact of a marginal change in parameter $b$ on utility along the transition path between two steady states. It decomposes the impact of the policy 
change into a cumulative change in capital from period 0 to period $t, \frac{d U}{d k}\left(\sum_{i=0}^{t-1} \psi_{k}(k ; b)^{i}\right) \psi_{b}(k ; b)$, an incremental effect, $\frac{\partial U}{\partial k_{t+1}} \psi_{k}(k ; b)^{t} \psi_{b}(k ; b)$, and the direct effect $\frac{\partial U}{\partial b}$ which is common to all cohorts born after period 0 .

Comparison of the key formulas (17), 18, and the definition of $\frac{d U}{d k}$ in Proposition 2 indicates that while (17) describes the full transition path, it contains only coefficients which are also present in (the limiting) formula (18), which describes the steady state effects only. That is, the evaluation of the transition path requires no additional information over what is necessary to carry out the steady state analysis.

Moreover, the series (17) in Proposition 2 is a useful screening device to examine whether a change in $b$ increases utility for all generations born after period 0 :

Corollary 1. A change in $b$ increases utility for all cohorts born in $t \geqq 0$ if $\frac{d U_{0}}{d b}>0$, and $\frac{d U_{s s}}{d b}>0$. If $\frac{d U_{0}}{d b}<0$ and $\frac{d U_{s s}}{d b}<0$, it reduces utility for all cohorts along the transition path.

Proof. see Appendix B

Corollary 1 shows that if utility for cohort 1 and steady state utility increase in $b$, then all cohorts benefit from the change in $b$ along the transition path. That is, due to the monotonous dynamics of utility, it suffices to check two derivatives to Pareto-evaluate the complete transition path.

It also follows from Proposition 2, and the implied monotonous dynamics of utility, that for cohorts born in $t \geqq 0$ there is an upper bound for the number of changes in signs of marginal utility that can occur along the transition path:

Corollary 2. Marginal utility $\frac{d U_{t}}{d b}$ changes signs at most once along the transition path. In the case where the sign of marginal utility changes, we have $\operatorname{sign}\left(\frac{d U_{0}}{d b}\right) \neq \operatorname{sign}\left(\frac{d U_{s s}}{d b}\right)$.

Proof. see Appendix B

\section{Application}

To illustrate Proposition 2 we revisit the well known steady state analysis of public debt by Diamond (1965), and show that the transitional dynamics associated with changes in the debt to labor ratio $b$ can easily be calculated. The main insight that emerges from this simple example is that changes in utility along the transition path (i) can overshoot long-run steady 
state utility, and (ii) can differ in sign from the steady state effects, even though both effects are calculated from the same coefficients.

With this example in place, we then briefly compare the present analytical approach to the alternative geometric argument which was developed by Matsuyama (1991) to study the transitional dynamics in OLG economies which do not alow for explicit solutions.

Following Diamond (1965) we incorporate a constant debt to labor ratio $b$ into our model (3)-(5), and obtain:

$$
\begin{aligned}
& \max _{s_{t}, c_{t}^{1}, c_{t+1}^{2}} U_{t}=U\left(c_{t}^{1}, c_{t+1}^{2}\right) \quad \text { s.t. } \quad c_{t}^{1}=w_{t}-\left(r_{t}-n\right) b-s_{t}, \quad c_{t+1}^{2}=s_{t}\left(1+r_{t+1}\right), \\
& \frac{U_{c^{1}}}{U_{c^{2}}}=1+r_{t+1} \quad \Leftrightarrow \quad s_{t}=s_{t}\left(w_{t}-\left(r_{t}-n\right) b, r_{t+1}\right), \quad 0<s_{w}<1, \quad s_{r} \gtreqless 0, \\
& (1+n)\left(k_{t+1}+b\right)=s_{t}\left(w_{t}-\left(r_{t}-n\right) b, r_{t+1}\right) \quad \Leftrightarrow \quad k_{t+1}=\psi\left(k_{t} ; b\right) .
\end{aligned}
$$

To apply Proposition 2 , respectively formula 17 , we calculate the coefficients $\psi_{k}, \psi_{b}, \frac{d U}{d k}, \frac{\partial U}{\partial k_{t+1}}, \frac{\partial U}{\partial b}$. As Diamond (1965), pp. 1132-1139, points out, it follows from (1), (2), and (26) that capital $k$ converges monotonically for the Walrasian economy:

$$
\psi_{k}(k ; b):=\frac{d k_{t+1}}{d k_{t}}=\frac{-s_{w} f^{\prime \prime}(k)(k+b)}{(1+n)-s_{r} f^{\prime \prime}(k)}, \quad 0<\psi_{k}(k ; b)<1,
$$

and increases in the debt to labor ratio $b$ reduce capital formation:

$$
\psi_{b}(k ; b):=\frac{d k_{t+1}}{d b}=\frac{-s_{w}(r-n)-(1+n)}{(1+n)-s_{r} f^{\prime \prime}(k)}<0 .
$$

Where $\psi_{b}<0$ in (28) follows from (27) 8 Finally, to calculate the remaining coefficients, we substitute (2) into (24), which yields

$$
U_{t}=U\left(f\left(k_{t}\right)-k_{t} f^{\prime}\left(k_{t}\right)-\left(f^{\prime}\left(k_{t}\right)-n\right) b-s, s \times\left(1+f^{\prime}\left(k_{t+1}\right)\right)\right) .
$$

Using (25) as an envelope for (29), we have around the steady state:

$$
\begin{aligned}
\frac{d U}{d k} & =\frac{\partial U}{\partial k_{t}}+\frac{\partial U}{\partial k_{t+1}}=-U_{c^{1}}\left(k+b-\frac{s}{1+r}\right) f^{\prime \prime}(k) \\
& =\mid 26]-U_{c^{1}}(r-n)(k+b) f^{\prime \prime}(k) \frac{1}{1+r} \\
\frac{\partial U}{\partial k_{t+1}} & =U_{c^{1}} \frac{s}{1+r} f^{\prime \prime}(k) ; \quad \frac{\partial U}{\partial b}=-U_{c^{1}}(r-n) .
\end{aligned}
$$

${ }^{8}$ To see this, note that the numerator in 28$)$ is negative, i.e., $-s_{w} r+\left(s_{w}-1\right) n-1<0$, since $r=f^{\prime}(k)>0$, $n>-1$ and $0<s_{w}<1$. Finally, the denominator is positive since the denominator $(1+n)-s_{r} f^{\prime \prime}(k)$ in 27$)$ must be positive. 
Substituting (30) and (31) into (17) yields the marginal change in utility for a cohort born $t$ periods after parameter $b$ was changed:

$$
\frac{d U_{t}}{d b}=-U_{c^{1}}(r-n)(k+b) f^{\prime \prime}(k)\left(\sum_{i=0}^{t-1} \psi_{k}^{i}\right) \psi_{b} \frac{1}{1+r}+U_{c^{1}} \frac{s}{1+r} f^{\prime \prime}(k) \psi_{k}^{t} \psi_{b}-U_{c^{1}}(r-n) .
$$

Factoring out and use of the life-cycle savings condition allows to rewrite marginal utility of cohort $t$ as

$$
\frac{d U_{t}}{d b}=-U_{c^{1}}(r-n)\left[\left(\sum_{i=0}^{t-1} \psi_{k}^{i}\right) \psi_{b}(k+b) f^{\prime \prime}(k) \frac{1}{1+r}+1\right]+U_{c^{1}}(k+b) \frac{1+n}{1+r} f^{\prime \prime}(k) \psi_{k}^{t} \psi_{b} .
$$

To calculate the steady state effect, we let $t \rightarrow \infty$ in $(32)$ :

$$
\frac{d U_{s s}}{d b}=-U_{c^{1}}(r-n)\left(\frac{1}{1-\psi_{k}} \psi_{b}(k+b) f^{\prime \prime}(k) \frac{1}{1+r}+1\right)
$$

and find that 33 reflects exactly the steady state interest rate condition $r \gtreqless n$ derived in Diamond (1965).

However, equations (32) and (33) also describe how increases in the debt to labor ratio affect utility of those households who live during the period of transition. Regarding the proceeds of increases in the debt $b$, we assume that they are allocated to the old generation -1 , which lives in retirement in period 0 when the change in $b$ is enacted. In the sequel, we do not record the changes in utility for generation -1 since they are trivial. From (32) and (33) we read the following propositions:

Proposition 3. If $r \leqq n$, a marginal increase of the debt to labor ratio $b$ in period 0 increases utility for all generations born in $t \geqq 0$. If the difference $n-r$ is small and the speed of convergence of the capital stock is high ( $\psi_{k}$ is small), such that $\frac{n-r}{1+n}<1-\psi_{k}$, then short-run gains are larger than long-run gains, that is, $U_{t}>U_{t+1}$ along the transition path.

Proof. With $r<n, 0<\psi_{k}<1$, and $\psi_{b}<0$, all expressions in (32) and (33) are positive. That is, increases in debt unambiguously increase utility for all cohorts. To derive the condition under which $U_{t}>U_{t+1}$, we note that by 32 we have $\frac{d U_{t}}{d b}-\frac{d U_{t+1}}{d b}=-U_{c^{1}}(k+b) \frac{1+n}{1+r} f^{\prime \prime}(k) \psi_{k}^{t} \psi_{b}(-$ $\left.\frac{(r-n)}{1+n}+\psi_{k}-1\right)$. Hence, if $\left(-\frac{(r-n)}{1+n}+\psi_{k}-1\right)<0$, or, equivalently, $0<\frac{n-r}{1+n}<1-\psi_{k}$, short-run gains are larger than long-run gains. Finally, we note that the difference $d_{t}:=\frac{d U_{t}}{d b}-\frac{d U_{t+1}}{d b}=$ $-U_{c^{1}}(k+b) \frac{1+n}{1+r} f^{\prime \prime}(k) \psi_{k}^{t} \psi_{b}\left(-\frac{(r-n)}{1+n}+\psi_{k}-1\right)$ converges monotonically since $0<\psi_{k}<1$.

Using similar arguments (32) and (33) also show that: 
Proposition 4. Suppose that $r-n>0$, and $r-n<\frac{(k+b) \frac{1+n}{1+r} f^{\prime \prime}(k) \psi_{k}^{T} \psi_{b}}{\left(\sum_{i=0}^{T-1} \psi_{k}^{i}\right) \psi_{b}(k+b) f^{\prime \prime}(k) \frac{1}{1+r}+1}$ for $a T>0$. In this case, marginal increases (decreases) of the debt to labor ratio $b$ in period 0 increase (decrease) utility for generations born between period 0 and period $T$. However, eventually, $b$ reduces (increases) utility for generations born after period $T$ and for those cohorts that live in the new steady state.

Put differently, Proposition 4 indicates that a reduction in the size of the debt, that is, a partial default on the old generation -1 in period zero, increases steady state utility. However, even though all generations born in $t \geqq 0$ are taxed less, such a policy change may lower utility for many cohorts during the period of transition. To see how a reduction in debt may decrease utility even though $r-n>0$, we multiply (32) with $d b<0$ and note that $d U_{t}<0$ obtains if the negative incremental effect $U_{c^{1}}(k+b) \frac{1+n}{1+r} f^{\prime \prime}(k) \psi_{k}^{t} \psi_{b} d b<0$, which describes how increased capital accumulation reduces the rate of return on savings, outweighs the beneficial cumulative effect, $-U_{c^{1}}(r-n)(k+b) f^{\prime \prime}(k)\left(\sum_{i=0}^{t-1} \psi_{k}^{i}\right) \psi_{b} \frac{1}{1+r} d b>0$, and direct effect $-U_{c^{1}}(r-n) d b>0$. However, as the economy approaches the new steady state, the incremental effect vanishes, and eventually, cumulative and direct effect dominate.

In contrast, for the Marshallian economy ${ }^{9}$ where $-1<\psi_{k}<0$, we have $\psi_{b}>0$, and 32 indicates:

Proposition 5. In an economy which grows on a path sufficiently close to the golden rule, such that there exists a $T>0$ for which we have $|r-n|<\left|\frac{(k+b) \frac{1+n}{1+r} f^{\prime \prime}(k) \psi_{k}^{T} \psi_{b}}{\left(\sum_{i=0}^{T-1} \psi_{k}^{i}\right) \psi_{b}(k+b) f^{\prime \prime}(k) \frac{1}{1+r}+1}\right|$, any change of the debt to labor ratio $b$ in period 0 reduces utility for every second generation born in $0<t<T$. If $r=n$, a change in public debt reduces utility for every second generation along the entire transition path.

Diagram 2 illustrates propositions 4 and 5 . Taken together, propositions 345 show that in economies which grow on a path sufficiently close to the golden rule there can be overshooting. That is, the changes in utility along the transition path may be algebraically larger than those obtained in the long-run. Moreover, these changes in utility along the transition path may differ in sign from the long-run effect.

\footnotetext{
${ }^{9}$ See Diamond (1965), p. 1132 and p. 1148, for the details on this case. At this point we exploit the analysis by Diamond (1965) and take the fact that $-1<\psi_{k}<0$ and $0<\psi_{b}$ (debt increases capital accumulation) from Diamond's analysis.
} 


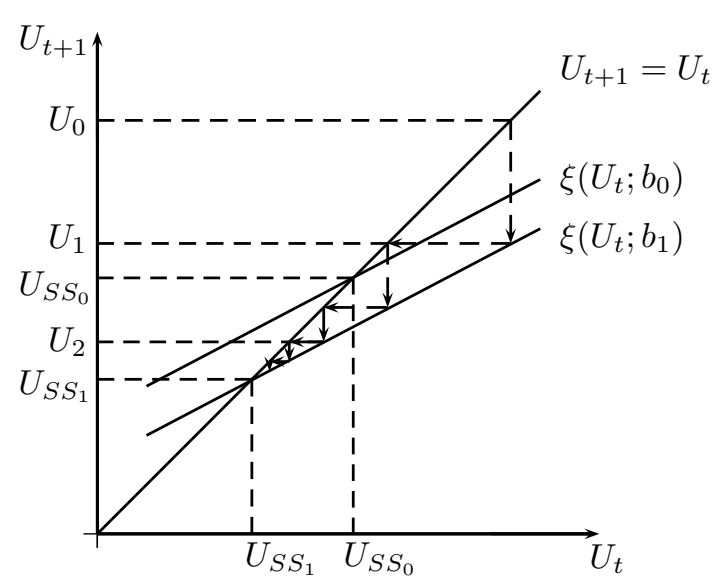

$2 a$

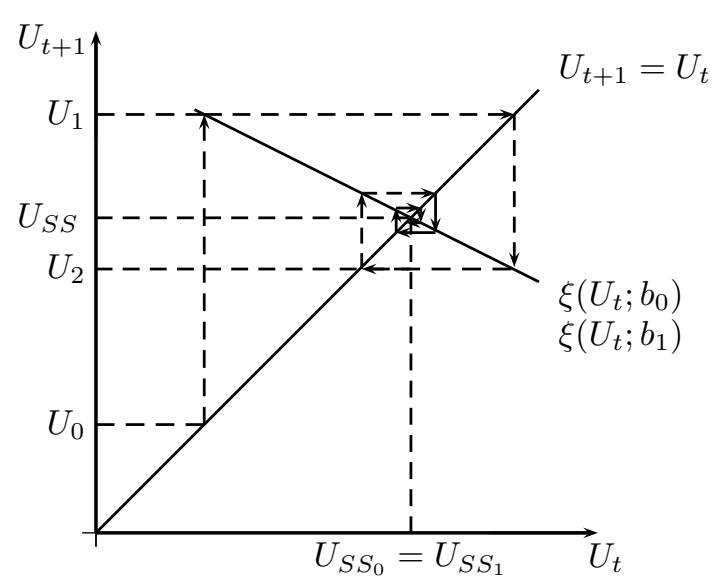

$2 b$

Diagram 2: Overshooting of Utility.

Diagram 2a illustrates an increase in public debt for $0<r-n<\varepsilon$ in the Walrasian economy, which increases utility for the first two cohorts due to the increased rate of return. However, utility falls for all cohorts born after period 1 . Diagram $2 b$ depicts a marginal increase in $b$ for the Marshallian economy which is in a steady state where $r=n$. In this case, the (first order) impact on steady state utility is zero. Along the transition path, however, the impact is negative (positive) for generations born in even (odd) periods.

Propositions 345, respectively equations 32 and (33), also show that the coefficients involved in the steady state comparison are identical to those required to study the transitional dynamics. Moreover, propositions 35 reveal that the implications of a given parameter change depend critically on the way in which the capital market converges towards equilibrium. In the case of the Marshallian economy, the competitive market system discards capital along the transition path so asymmetrically that a simple change in the debt to labor ratio rarely leads to Pareto-improvements, even if the economy is growing on an inefficient path where $r<n$. On the contrary, the market process in the Walrasian case is so well behaved that a simple increase in the size of the debt can be Pareto-improving. Moreover, if the economy is close to a golden rule allocation, such a change in debt will benefit those cohorts that live during the period of transition more than those who live in the long-run equilibrium. However, according to Proposition 4, a permanent reduction of the debt in period 0 has ambiguous effects on utility along the transition path even if $r>n$ in the economy's initial steady state.

The key difference between the present analytical approach and the geometric argument developed by Matsuyama (1991) lies in the ease of use of the present argument, since it only requires knowledge of the steady state coefficients, which are usually available. On the contrary, the analysis by Matsuyama (1991) is demanding in that it requires repeated drawing of several 
curves, which are intertemporally entangled. Moreover, as Matsuyama (1991), p. 261, points out, the geometric approach works best for economies where parameter changes do not shift the indifference curves in the $w, r$ plane. While the adoption of more productive technologies studied by Matsuyama (1991) satisfies this condition, the problem of changing public debt does not.10 The same applies in general to policy parameters that affect the households' budget constraints directly. The present approach is therefore less restrictive. Finally, the use of an analytical argument makes it easier to work consistently with the formal assumptions placed on technology and preferences.

\section{Conclusion}

The normative evaluation of the neoclassical OLG model with productive capital is usually either confined to pure steady state comparisons, or to economies that rely on rather restrictive assumptions which allow to calculate transition paths explicitly. The method developed here overcomes this difficulty. It is not more complicated than a pure steady state analysis. At the same time it allows to characterize the utility implications of one-time permanent parameter changes along the entire transition path between two steady states. Moreover, since an explicit solution of the transition path is not required, the present method can be applied to general economies which do not rely on the restrictive assumptions that are otherwise necessary to solve for transition paths explicitly.

We have chosen the well known steady state analysis by Diamond (1965) to illustrate how available knowledge of steady state coefficients $\psi_{k}$ and $\psi_{b}$ allows to characterize the associated transition paths. However, the method developed here can equally be applied to other studies which also confine attention to steady states only. ${ }^{11}$ While it adds the complete transition path which was omitted so far in these studies, it does not require any additional assumptions or computations. Moreover, even though the transition path's evaluation is simple, it provides

\footnotetext{
${ }^{10}$ Changes in public debt pivot the indifference curve in the wage interest plane around the point where $r=n$. To see this, recall that $\frac{\partial U(w-(r-n) b-s,(1+r) s)}{\partial b}=-U_{c^{1}}(r-n)$. Hence, $\left.\frac{d w}{d b}\right|_{d U=0}=r-n \gtreqless 0$, and $\left.\frac{d r}{d b}\right|_{d U=0}=\frac{r-n}{b-\frac{s}{1+r}} \gtreqless 0$. That is, the set of $w, r$ pairs, which yield constant utility, must change with varying levels of $b$ as long as $r \neq n$.

${ }^{11}$ Representative examples where the coefficients $\psi_{k}$ and $\psi_{b}$ are available but the normative analysis is confined to steady states only, are Diamond (1970), Samuelson (1975), Galor and Polemarchakis (1987), Galor (1988), or Casarico and Devillanova (2008).
} 
interesting economic insights. Namely, it shows that changes in utility along the transition path tend to be algebraically larger than long-run effects if the economy converges rapidly towards the new steady state, or if the economy grows on a path which is sufficiently close to the golden rule level. More importantly, however, we have derived the precise conditions under which changes in utility along the transition path differ in sign from those changes that obtain in the long-run. These conditions can be used to decide whether a given parameter change can bring about Pareto-improvements. That is, unlike previous studies that rely on steady state comparisons, the present method allows to Pareto-evaluate parameter changes.

Finally, to interpret the interaction between changes in policy parameters and the competitive incomplete market system, we compared Marshallian and Walrasian economies. This comparison showed that given parameter changes, which are Pareto-improving for the monotonous Walrasian economy, are not necessarily Pareto-improving in the Marshallian economy where utility converges in cobweb manner towards its steady state value. 


\section{A Construction of $\psi(), \phi()$, and $\xi()$}

We recall the implicit function theorem ${ }^{12}$

Proposition 6. For a continuously differentiable function $F(x, y)=0$ with a partial derivative $F_{y} \neq 0$, there exists locally a continuously differentiable function $g()$ such that $y=g(x)$, and $\frac{d y}{d x}=g^{\prime}(x)=-\frac{F_{x}}{F_{y}}$. If the derivative $F_{x}$ is also non-vanishing, $g()$ has a continuously differentiable inverse function $g^{-1}()$ such that $x=g^{-1}(y)$, and $\frac{d x}{d y}=g^{-1^{\prime}}(y)=-\frac{F_{y}}{F_{x}}$.

To apply theorem 6, we note that it follows directly from our assumptions that all primitive functions $U(),, s(),, f^{\prime}(), w()$, and $r()$, in (9) and (10) are at least once continuously differentiable 13 Moreover, we know that the composition of continuously differentiable functions is continuously differentiable. Hence, to show the existence of $\phi(), \phi^{-1}(), \psi()$, and $\psi^{-1}()$ by means of Proposition 6, it only remains to check that the derivatives $\frac{d k_{t+1}}{d k_{t}}=\psi^{\prime}(k), \frac{d k_{t}}{d k_{t+1}}=\psi^{-1^{\prime}}(k)$, $\frac{d k_{t}}{d U_{t}}=\phi^{\prime}(U)$, and $\frac{d U_{t}}{d k_{t}}=\phi^{-1^{\prime}}(k)$, are well defined around a steady state, where $k_{t}=k_{t+1}=k$. With these functions in place, the properties of $\xi()=\phi^{-1}(\psi(\phi()))$ in 111) and its inverse $\xi^{-1}()=\phi^{-1}\left(\psi^{-1}(\phi())\right)$ follow at once.

1) Derivation of $\psi()$ and $\psi^{-1}()$ : To show that an implicit function $\psi()$ which satisfies

$$
(1+n) k_{t+1}=s\left(w\left(k_{t}\right), r\left(k_{t+1}\right)\right) \quad \Leftrightarrow \quad k_{t+1}=\psi\left(k_{t}\right), \quad \psi \in \mathcal{C}^{1}
$$

exists, we note that the implicit derivative $\frac{d k_{t+1}}{d k_{t}}=\psi^{\prime}(k)=\frac{-s_{w} f^{\prime \prime}(k)}{(1+n)-s_{r} f^{\prime \prime}(k)}$ is, locally, well defined. This, follows immediately from assumption 77 , which ensures that $0<\frac{-s_{w} f^{\prime \prime}(k)}{(1+n)-s_{r} f^{\prime \prime}(k)}<1$. Moreover, all functions in this fraction are at least continuous. Hence, according to Proposition 6 there exists a function $\psi()$, such that $k_{t+1}=\psi\left(k_{t}\right)$. Finally, since $-s_{w} f^{\prime \prime}(k) \neq 0$, the inverse function $\psi^{-1}()$ exists and has the derivative $\psi^{-1^{\prime}}(k)=\frac{(1+n)-s_{r} f^{\prime \prime}(k)}{-s_{w} f^{\prime \prime}(k)}>0$.

2) Derivation of $\phi()$ and $\phi^{-1}()$ : Recalling the household problem (3) and (4) we have

$$
\begin{aligned}
U_{t} & =U\left(w\left(k_{t}\right)-s\left(w\left(k_{t}\right), r\left(k_{t+1}\right)\right),\left(1+r\left(k_{t+1}\right)\right) s\left(w\left(k_{t}\right), r\left(k_{t+1}\right)\right)\right)=U\left(k_{t}, k_{t+1}\right) \\
& =\mid 96\left(k_{t}, \psi\left(k_{t}\right)\right) \Leftrightarrow \quad k_{t}=\phi\left(U_{t}\right), \quad \phi \in \mathcal{C}^{1} .
\end{aligned}
$$

\footnotetext{
${ }^{12}$ See, e.g., Lee (2003) pp. 164-166, for a proof of the implicit function theorem and the inverse function theorem.

${ }^{13}$ In particular, the savings function implied by the euler equation (4) is once continuously differentiable since $U($,$) is by assumption twice continuously differentiable.$
} 
To understand $\phi()$ in A.2), it is useful to start with the derivative of its inverse:

$$
\phi^{-1^{\prime}}(k)=\frac{d U_{t}}{d k_{t}}=\frac{\partial U}{\partial k_{t}}+\frac{\partial U}{\partial k_{t+1}} \psi^{\prime}(k) .
$$

Using the euler equation (4) as an envelope, we rewrite (A.3) such that

$$
\begin{aligned}
\phi^{-1^{\prime}}(k) & =\frac{d U_{t}}{d k_{t}}=U_{c^{1}}\left(-f^{\prime \prime}(k) k+\psi^{\prime}(k) f^{\prime \prime}(k) \frac{s}{1+r}\right) \\
& ={ }_{A .1}-U_{c^{1}}\left(k-\psi^{\prime}(k) \frac{(1+n) k}{1+r}\right) f^{\prime \prime}(k), \\
& =-U_{c^{1}}\left((1+r)-\psi^{\prime}(k)(1+n)\right) \frac{1}{1+r} f^{\prime \prime}(k) k .
\end{aligned}
$$

Hence, $\phi^{-1^{\prime}}(k)$ is continuous and bounded for (positive) finite capital intensities, which are ensured by the standard existence and uniqueness conditions (6) and (7).

However, A.4 may be zero. In this case, we have $\frac{d U_{t}}{d k_{t}}=0$, and changes in the capital intensity do not change utility. That is, in this special case, utility does not change as the capital stock converges towards the steady state level, and $\phi()$ is not well defined ${ }^{14}$ Naturally, however, the dynamics of utility are trivial in this case and require no evaluation ${ }^{15}$ In all cases of interest, where utility changes over time, $\phi()$ is well defined and we have:

$$
\phi^{\prime}(U)=\frac{d k_{t}}{d U_{t}}=\frac{1}{\left(-U_{c^{1}}\left((1+r)-\psi^{\prime}(k)(1+n)\right) \frac{1}{1+r} f^{\prime \prime}(k) k\right)} .
$$

3) Derivation of $\xi()$ and $\xi^{-1}()$ : Taken together, paragraphs 1 and 2 show that, as long as utility changes over time, the function $\xi()=\phi^{-1}(\psi(\phi()))$ in (11) is well defined. In particular, since $\psi(), \psi^{-1}(), \phi()$ and $\phi^{-1}()$ are all continuously differentiable functions, $\xi()$ and $\xi^{-1}()=\phi^{-1}\left(\psi^{-1}(\phi())\right)$ are also both continuously differentiable functions. Hence, $\xi()$ is a $\mathcal{C}^{1}$ diffeomorphism. The difference equation $U_{t+1}=\xi\left(U_{t}\right)$ is therefore symmetric with respect to time. That is, we can write $U_{t-1}=\xi^{-1}\left(U_{t}\right)$ and use current utilities $U_{t}$ to calculate past utilities $U_{t-1}$.

\footnotetext{
${ }^{14}$ That is, $\phi()$ only allows to exchange coordinates $k_{t}$ and $U_{t}$ if $k_{t}$ and $U_{t}$ are related.

${ }^{15}$ We may note that A.4 implies that this special case, where utility does not change with capital, is only possible for the Walrasian economy which is dynamically inefficient $(r<n)$ such that $0<\frac{1+r}{1+n}=\psi^{\prime}(k)<1$. This condition is naturally always violated in the Marshallian economy where $\psi^{\prime}(k)<0$.
} 


\section{B Proof of Corollaries 1 and 2}

\subsection{Corollary 1:}

Proof. Defining $A:=\frac{d U}{d k} \psi_{b}, B:=\frac{\partial U}{\partial k_{t+1}} \psi_{b}$, and $C:=\frac{\partial U}{\partial b}$, we have

$$
\begin{aligned}
& \frac{d U_{0}}{d b}=0+B+C>0 \quad \Leftrightarrow \quad-C<B, \\
& \frac{d U_{1}}{d b}=A+B \psi_{k}+C, \\
& \frac{d U_{2}}{d b}=A\left(1+\psi_{k}\right)+B \psi_{k}^{2}+C, \\
& \cdot \\
& \frac{d U_{s s}}{d b}=A \frac{1}{1-\psi_{k}}+C>0 \quad \Leftrightarrow \quad-C\left(1-\psi_{k}\right)<A .
\end{aligned}
$$

We prove corollary 1 in two steps: 1) first we show that (B.1) and (B.4) imply that (B.2) is positive, i.e., $\left.\frac{d U_{1}}{d b}=A+B \psi_{k}+C>0 ; 2\right)$ in a second step we show that the series $\frac{d U_{t}}{d b}$, with $t \geqq 1$, evolves monotonically and has a positive lower bound $\frac{d U_{s s}}{d b}>0$. Taken together, it follows from these steps that if $\frac{d U_{0}}{d b}$ and $\frac{d U_{s s}}{d b}$ are both positive, all derivatives $\frac{d U_{t}}{d b}$ with $t \geqq 1$ are positive, which is what we need to show.

Step 1 To show that $(\mathrm{B} .1)$ and $(\overline{B .4})$ imply that $(\overline{B .2})$ is positive, we recall that $0<\psi_{k}<1$ and distinguish eight cases involving the various possibilities $A \gtrless 0, B \gtrless 0, C \gtrless 0$ :

1. $A>0, B>0, C<0$ : We have to show that $A+B \psi_{k}+C>0$. To do so, we write $A+B \psi_{k}+C>$ B.1]B.4 $\left(1-\psi_{k}\right)(-C)+\psi_{k}(-C)+C=0$.

2. $A>0, B>0, C>0$ : Clearly, $A+B \psi_{k}+C>0$.

3. $A>0, B<0, C<0$ : In this case we have $B+C<0$. This violates our assumption (B.1).

4. $A>0, B<0, C>0$ : We have $A+B \psi_{k}+C>B \psi_{k}+C>B+C>\mid[B .1 \mid 0$.

5. $A<0, B>0, C<0$ : Would violate assumption (B.4).

6. $A<0, B>0, C>0$ : We show that $A+B \psi_{k}+C>0$ by contradiction. Assuming the contrary, $A+B \psi_{k}+C<0$, yields $A+B \psi_{k}<-C<\mid B .4 \frac{A}{\left(1-\psi_{k}\right)}$. Hence, $A<$ 
$\frac{A}{\left(1-\psi_{k}\right)}-B \psi_{k}<\frac{A}{\left(1-\psi_{k}\right)}$. However, $A<\frac{A}{\left(1-\psi_{k}\right)}$ is a contradiction since $\frac{1}{\left(1-\psi_{k}\right)}>1$ and $A<0$ imply $A>\frac{A}{\left(1-\psi_{k}\right)}$.

7. $\left.A<0, B<0, C>0: A+B \psi_{k}+C=A-(-B) \psi_{k}+C>B .1\right]-\left(1-\psi_{k}\right) C-C \psi_{k}+C=0$.

8. $A<0, B<0, C<0$ : Violates assumptions (B.1) and (B.4).

Taken together, cases 1-8 show that if $(\mathrm{B} .1)$ and $(\mathrm{B} .4)$ are positive, then $(\mathrm{B} .2)$ is also positive.

Step 2 It remains to show that $\frac{d U_{t}}{d b}$ evolves monotonically over time. From 17) we have $\frac{d U_{t+1}}{d b}-\frac{d U_{t}}{d b}=\psi_{k}^{t}\left(A+B\left(\psi_{k}-1\right)\right)$. Hence, $\frac{d U_{t}}{d b}$ is monotonically increasing or decreasing. Consequently, since $\frac{d U_{t}}{d b}$ is positive at $t=1$, it increases further over time if $A+B\left(\psi_{k}-1\right)>0$. If $A+B\left(\psi_{k}-1\right)<0$, it decreases over time but has a positive lower bound since $\frac{d U_{\mid s s}}{d b}>0$. Hence, in both cases, all derivatives $\frac{d U_{t}}{d b}$ must be positive for $t \geqq 0$, which concludes our proof.

Finally, we note that all foregoing steps can also be performed if (B.1), (B.4), and $A, B, C$ involve weak inequalities.

\subsection{Corollary 2}

Proof. Since the proof of corollary 2 is parallel to the proof of corollary 1 , we only present a short sketch. In a first step we define $A:=\frac{d U}{d k} \psi_{b}, B:=\frac{\partial U}{\partial k_{t+1}} \psi_{b}, C:=\frac{\partial U}{\partial b}$, and distinguish 8 cases involving $A \gtrless 0, B \gtrless 0, C \gtrless 0$. For all 8 cases it is straightforward to show that $\frac{d U_{0}}{d b}>0$ and $\frac{d U_{1}}{d b}<0$ imply that $\frac{d U_{s s}}{d b}<0$. Moreover, due to the monotonicity of the sequence $\frac{d U_{t}}{d b}$, a change in signs at a second point in time $t \geqq 1$ is not possible. Hence, if $\frac{d U_{0}}{d b}>0$ and $\frac{d U_{1}}{d b}<0$, then marginal utility changes signs only once between cohort 0 and cohort 1 . The same argument applies when $\frac{d U_{0}}{d b}<0$ and $\frac{d U_{1}}{d b}>0$.

If we assume that $\frac{d U_{0}}{d b}>0$ and $\frac{d U_{1}}{d b}>0$, we may have for $A<0, B>0, C<0$, or, $A>0, B>0, C<0$, that $\frac{d U_{\mid s s}}{d b}<0$. In this case, marginal utility changes signs once at some $T>1$.

Moreover, we note that exchanging $>$ for $<$, as well as changing strict inequalities with weak inequalities in the foregoing calculations, yields the same conclusions, namely, that marginal utility can change signs at most once.

Finally, it follows from corollary 1 that marginal utility cannot change signs if $\operatorname{sign}\left(\frac{d U_{0}}{d b}\right)=$ $\operatorname{sign}\left(\frac{d U_{s s}}{d b}\right)$. 


\section{References}

Casarico, A. and Devillanova, C. (2008). Capital-skill complementarity and the redistributive effects of social security reform. Journal of Public Economics, 92:672-683.

de la Croix, D. and Michel, P. (2002). A Theory of Economic Growth. Cambridge: Cambridge Univ. Press.

Diamond, P. A. (1965). National debt in a neoclassical growth model. American Economic Review, 55(5):1126-1150.

Diamond, P. A. (1970). Incidence of an interest income tax. Journal of Economic Theory, $2: 211-224$.

Galor, O. (1988). The long-run implications of a Hicks-neutral technical progress. International Economic Review, 29(1):177-183.

Galor, O. and Polemarchakis, H. (1987). Intertemporal equilibrium and the transfer paradox. Review of Economic Studies, 54(1):147-156.

Galor, O. and Ryder, H. (1989). Existence, uniqueness and stability of equilibrium in an overlapping-generations model with productive capital. Journal of Economic Theory, 49(2):360-375.

Lee, J. M. (2003). Introduction to Smooth Manifolds. Graduate Texts in Mathematics 218; Springer Verlag.

Matsuyama, K. (1991). Immiserizing growth in Diamonds overlapping generations model: A geometrical exposition. International Economic Review, 32(1):251-262.

Michel, P. and de la Croix, D. (2000). Myopic and perfect foresight in the olg model. Economics Letters, 67:53-60.

Samuelson, P. A. (1941). The stability of equilibrium: Comparative statics and dynamics. Econometrica, 9(2):97-120.

Samuelson, P. A. (1975). The optimum growth rate for population. International Economic Review, 16(3):531-538.

von Weizsäcker, C. C. (1971). Steady State Capital Theory. Springer Verlag. 